Ann. Zootech., I962, 11 (4), 295-309.

\title{
INFLUENCE DE L'INTRODUCTION DE GRAISSE (SAINDOUX) DANS LA RATION SUR LA PRODUCTION ET LA COMPOSITION DU LAIT DE TRUIE
}

\author{
E. SAIMON-LEGAGNEUR \\ avec la collaboration technique de J. Rettagliati, A. Grude, Hélène Moutei, \\ Colette BaUdrillart et la participation de C. ZanNetos \\ Station de Recherches sur l'Élevage des Porcs, \\ Centre national de Recherches zootechniques, Jouy-en-Josas (Seine-et-Oise)
}

\section{SOMMAIRF}

Une expérience portant sur 40 truies Large White, réparties en 4 lots, a été entreprise pour étudier l'effet sur la production laitière de l'incorporation de I 7 p. Ioo de saindoux au régime de lactation.

On observe une augmentation d'environ ${ }_{5}$ p. roo de la quantité de lipides sécrétés dans le lait, qui apparaît même lorsque le régime est isoénergétique avec celui d'animaux témoins. On note, en outre, une certaine modification de la répartition des acides gras du lait (augmentation des acides insaturés) et une diminution de la perte de poids corporel au cours de la lactation.

Le poids au sevrage des porcelets est augmenté d'environ io p. Ioo.

\section{INTRODUCTION}

I1 est souvent difficile de faire ingérer à une truie en lactation la quantité d'aliment qui conviendrait à ses besoins. En ce qui concerne l'énergie notamment, il n'est plus possible, dès que la production laitière journalière excède $6 \mathrm{~kg}$ de lait, de couvrir les dépenses par la seule ingestion d'un aliment concentré du type classique (LobGE, 196r). Ceci comporte plusieurs conséquences : tout d'abord la truie doit prélever sur ses propres tissus les nutriments qui lui sont nécessaires pour couvrir ses besoins de lactation. Il s'ensuit une perte de poids souvent importante que l'animal doit combler par la suite et surtout une délipidation qui peut atteindre un degré considérable (SALMon-LEGAGNeur, Ig6I b). Enfin il n'est pas impossible que la truie limite alors sa production, en qualité et en quantité, à un niveau inférieur à celui qui pourrait être réalisé si sa ration était suffisante (SALMON-IEGAGNEUR, I957 ; LODGE, I959). 
L'une des solutions auxquelles on peut penser pour remédier à cet état de choses réside dans l'incorporation dans la ration, de substances plus riches en énergie que les aliments usuels, et principalement, des graisses.

Peu d'expériences ont été entreprises jusqu'à présent sur l'influence des graisses sur la production laitière des Truies. Mais, depuis que WIrETTT (I946), NorMaN (I955) et nous-mêmes (I960) ont montré que l'adjonction des différentes graisses animales au régime pouvait provoquer des modifications importantes du taux de lipides du lait de Truie, on est venu à se demander si celle-ci ne pourrait constituer un facteur d'amélioration de la production laitière et, au-delà, de la croissance des porcelets.

Nous avons entrepris dans ce but une expérience portant sur 40 truies, pour étudier l'influence d'un régime contenant $17 \mathrm{p}$. Ioo de saindoux sur la quantité de lait produite, la composition du lait et plus particulièrement la composition en acides gras, la croissance des porcelets et les variations de poids des mères. L'influence de ce régime a été testée par rapport à 11 r régime sans graisse dans les trois cas suivants :

$I^{\circ}$ avec un niveau isoazoté,

$2^{\circ}$ avec un rapport énergie/protéine égal,

$3^{\circ}$ avec un niveau isoénergétique et isoazoté.

\title{
MATÉRIEI, ET TECHNIQUES
}

\author{
a) Animaux. Mesures effectuées
}

4 lots de to truies de race Yorkshire Large White ont été constitués avec des animaux provenant de notre troupeau expérimental. Les truies avaient toutes déjà accompli au moins une lactation et reçu au cours de leur gestation une alimentation équilibrée convenable. Jee poids moyen, les antécédents et les résultats de parturition étaient identiques pour chacun de ces lots.

La lactation durait 8 semaines. Pendant toute la durée de l'expérience, les animaux étaient logés en cases individuelles dans une porcherie conditionnée $\left(20^{\circ} \mathrm{C}, \mathrm{l}: 55 \mathrm{p}\right.$. 100) et recevaient une ration journalière constante, exactement mesurée, sous forme d'une pâtée humide (1/3 aliment, 2/3 eau) répartie en deux repas égaux.

A la naissance, les portées étaient égalisées à 9 porcelets. Pendant la lactation, ces derniers avaient libre accès à un aliment de type courant pour porcelets.

Une fois par semaine, la production laitière de chaque truie était déterminée et un échantillon de lait prélevé pour l'analyse (SALmon-Legagneur, I959a). Le poids des truies, la croissance des porcelets et l'épaisseur du tissu adlipeux sous-cutané dorsal (déterminée aux ultra-sons) faisaient également l'objet de mesures hebdomadaires à heures fixes.

En outre, sur 5 truies de chaque lot, on a déterminé la digestibilité apparente des aliments (énergie, protéines) par la méthode à l'oxyde de chrome. Pour cela i p. roo en poids sec d'oxyde de chrome était mélangé soigneusement à la ration et, après une période de mise en régime de 5 jours, on prélevait sur la totalité des fèces quotidiennes un échantillon moyen journalier pendant une période de ro jours. La comparaison des teneurs moyennes, pour la période considérée, en oxyde de chrome dans l'aliment et les excreta permettait de connaître la digestibilité du régime.

\section{b) Régimes alimentaires}

Quatre régimes ont été utilisés, correspondant chacun à un lot expérimental :

I : Régime de base sans graisse ;

II : Régime de base + saindoux (isoazoté +20 p. ıoo calories) ;

III : Régime de base + saindoux + farine de poisson $(+20 \mathrm{p}$. 100 azote $+20 \mathrm{p}$. 100 calories, même rapport énergie/ $\mathrm{N}$ (que I).

IV : Régime II réduit de 20 p. ıoo(isoazoté, isoénergétique, mais 30 p. Ioo de l'énergie provient des lipides). 
Pour parvenir à ce résultat, on a remplacé une partie de l'amidon du régime de base par le saindoux, ou la farine de poisson (tableau r).

\section{TABLEAU I}

Constitution des régimes

\begin{tabular}{|c|c|c|c|c|}
\hline Régimes $\ldots \ldots \ldots \ldots \ldots \ldots \ldots \ldots \ldots \ldots \ldots \ldots$ & I & II & III & IV \\
\hline \multicolumn{5}{|l|}{ Constituants : } \\
\hline Blé .. & 50 & 50 & 55 & 55 \\
\hline Farine de luzerne ..... & 10 & 10 & 10 & 10 \\
\hline Tourteau de soja $\ldots \ldots \ldots \ldots \ldots \ldots \ldots \ldots \ldots \ldots$ & 7 & 7 & 7 & 7 \\
\hline 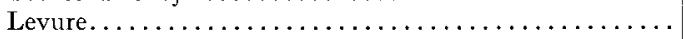 & 4 & 4 & 4 & 4 \\
\hline Farine de poisson $\ldots \ldots \ldots \ldots \ldots \ldots \ldots \ldots \ldots \ldots \ldots \ldots \ldots \ldots \ldots \ldots$ & 5 & 5 & 8 & 8 \\
\hline 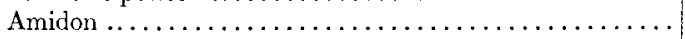 & 20 & 3 & & \\
\hline 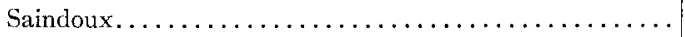 & & 17 & 17 & 17 \\
\hline \multirow[t]{2}{*}{ 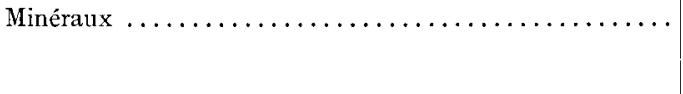 } & 4 & $l_{4}$ & 4 & 4 \\
\hline & 100 & 100 & 100 & 100 \\
\hline
\end{tabular}

Les aliments avaient les caractéristiques suivantes :

Matières azotées totales $(\mathrm{N} \times 6,25)$ p. 100

$\begin{array}{cccc}\text { I } & \text { II } & \text { III } & \text { IV } \\ \overline{17,51} & -17,45 & 20,53 & 20,50 \\ 3850 & 4840 & 4730 & 4770\end{array}$

Les rations journalières distribuées étaient les mêmes pour les 3 premiers lots : 4,2 kg d'aliment $\mathrm{sec}$; le 4 e lot recevait une ration plus faible de $20 \mathrm{p}$. Ioo, soit $3,5 \mathrm{~kg}$.

\section{c) Méthodes d'analyse}

L'azote des aliments était dosé selon la méthode Kjeldhal et les valeurs calorifiques étaient déterminées à la bombe calorimétrique de Ferry. L'oxyde de chrome était dosé après fusion alcaline et par dosage du pouvoir oxydant en présence d'iodure de potassium (RAYMOND et Minson, I955).

Les principaux constituants du lait (matières azotées, lipides, lactose, cendres) étaient dosés selon nos techniques habituelles (SALMON-LEGAGNEUR, I959 $b$ ).

Les acides gras polyéniques étaient dosés spectrophotométriquement après isomérisation alcaline (PAQUOT et al., 1962). La teneur en acide oléique était calculée après détermination de l'indice d'iode et celle en acides gras saturés par différence.

\section{RÉSULTATS}

\section{Io Digestibilité}

Le tableau 2 indique les coefficients moyens de digestibilité apparente que nous avons trouvés pour l'azote et l'énergie de chaque aliment. A partir de ces coefficients, nous avons également calculé les quantités de matière azotée et d'énergie digestibles ingérées. 
Comme on peut le constater, la digestibilité apparente a été assez peu affectée par la présence de graisse dans certains régimes, surtout en ce qui concerne la digestibilité de la matière azotée. Toutefois, il semble que l'énergie totale ait été un gen moins bien digérée dans les régimes contenant du saindoux (différence non significative).

Il en a résulté que les consommations d'azote digestible ont été les mêmes dans les lots I, II et IV et plus élevées de 20 p. Ioo dans le lot III. Comme, par ailleurs, les quantités de calories digestibles consommées par les lots II et III ont été supérieures de $\mathrm{r} 9,5 \mathrm{p}$. Ioo à celles du lot I, les lots I et III ont eu à leur disposition, comme nous le désirions, des aliments de même rapport calories/M. A. D. Par contre, le lot IV a disposé d'un peu moins de calories que prévu ( $3 \mathrm{p}$. Ioo de moins que le lot I).

TABLEAU 2

Coefficients de digestibilité

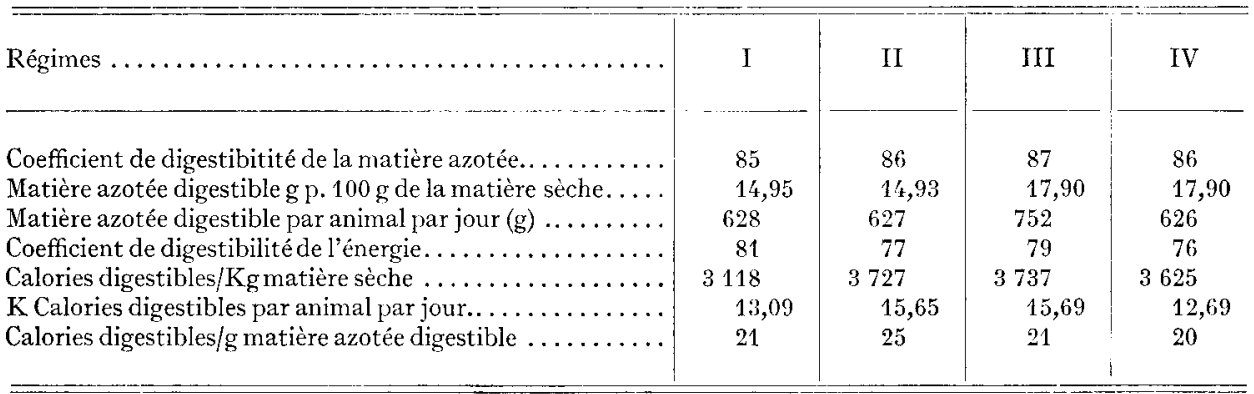

\section{$2^{\circ}$ Quantités de lait produites}

Les quantités totales de lait produites en moyenne par les truies de chaque lot, au cours des 8 semaines de lactation, ont été les suivantes :

\begin{tabular}{|c|c|}
\hline 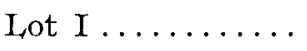 & $302 \pm 33$ \\
\hline Lot II & $33 I \pm 28$ \\
\hline I . & $293 \pm$ \\
\hline$\ldots \ldots \ldots$ & $295 \pm$ \\
\hline
\end{tabular}

Seules, les truies du lot II, qui recevaient un supplément de saindoux seul, ont produit un peu plus de lait que les autres (différence significative à $\mathrm{P}=0,05$ ). Par contre, celles du lot III, qui reçevaient autant d'énergie et davantage d'azote, ont produit moins de lait que ces dernières.

L'évolution des quantités produites au cours de la lactation est indiquée à la figure I et ne présente pas de caractère spécial.

\section{$3^{\circ}$ Composition du lait}

Le tableat 3 rapporte, pour chaque lot, la teneur moyenne du lait en ses principaux constituants et les figures 2 à 5 l'évolution de ces constituants au cours de la lactation. 
Les différences les plus importantes concernent les teneurs en lipides : les truies des lots recevant du saindoux ont produit un lait contenant Io à $I_{5} \mathrm{p}$. Ioo de graisse de plus que celles du lot I.

La figure 2 montre que ce résultat a été obtenu surtout en fin de lactation. Au début de la lactation, les taux de lipides sont semblables dans les 4 lots, mais alors

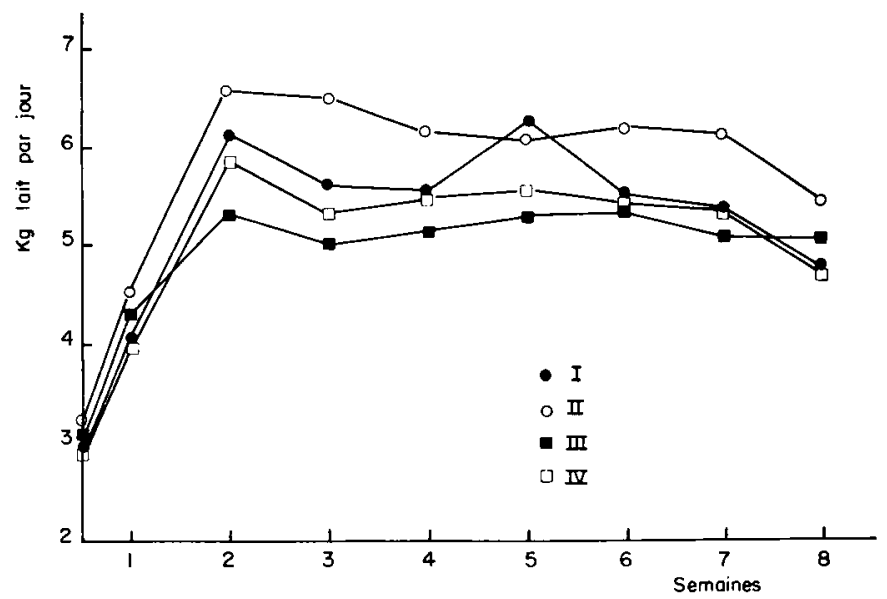

Fig. I. - Productions laitières

que, dès la $2^{\mathrm{e}}$ semaine, le taux de matière grasse du lot I baisse d'une façon classique, celui des trois lots recevant de la graisse se maintient à une valeur anormalement élevée.

\section{TABIEAU 3}

Composition chimique du lait ( $g$ p. $100 \mathrm{ml}$ de lait frais)

\begin{tabular}{|c|c|c|c|c|c|}
\hline Constituants & Eau & Mat. azotée & Liprides & Lactose & Cendres \\
\hline Lots : & & & & & \\
\hline$I \ldots \ldots \ldots$ & $80,4 \pm 1,1$ & $5,9 \pm 0,3$ & $7, \mathrm{t} \pm 0,2$ & $5,6 \pm 0,2$ & $0,89 \pm 0,03$ \\
\hline II $\ldots \ldots \ldots$ & $80,0 \pm 0,9$ & $5,9 \pm 0,4$ & $7,8 \pm 0,2$ & $5,4 \pm 0,2$ & $0,96 \pm 0,04$ \\
\hline III & $79,3 \pm 0,8$ & $6,2 \pm 0,3$ & $8,2 \pm 0,2$ & $5,3 \pm 0,2$ & $1,02 \pm 0,08$ \\
\hline IV ..... & $79,0 \pm 0,8$ & $6,1 \pm 0,3$ & $8,2 \pm 0,2$ & $5,2 \pm 0,2$ & $1,02 \pm 0,04$ \\
\hline
\end{tabular}

Si les teneurs moyennes en matière azotée ne révèlent aucune différence notable entre les lots, on peut remarquer, par contre, que le taux de lactose est légèrement plus élevé (non significativement) dans le lot témoin et diminue moins vite au cours de la lactation que dans les trois autres lots (figure 4). Inversement, la teneur en minéraux totaux est significativement plus élevée dans le lait des truies supplémentées en graisse (figure 5). 


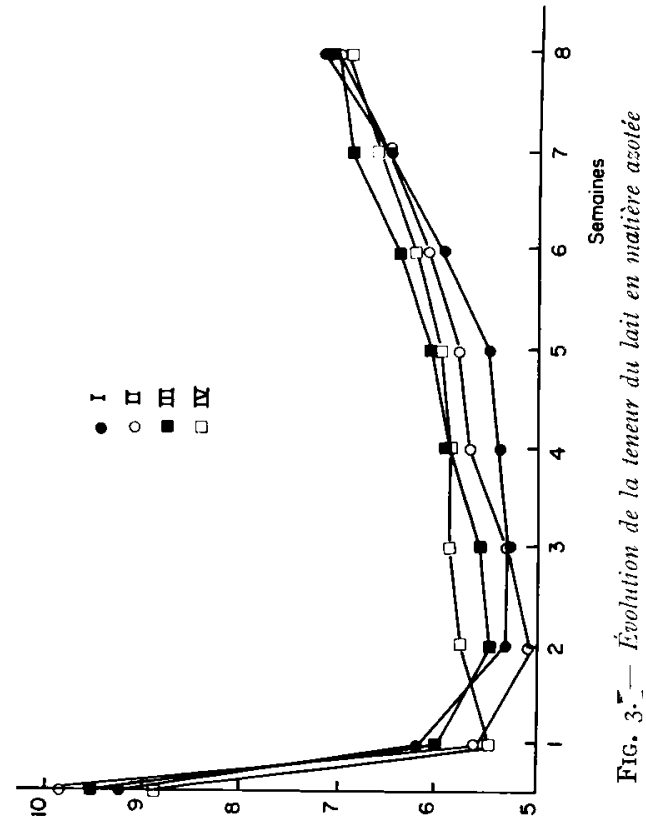

00106

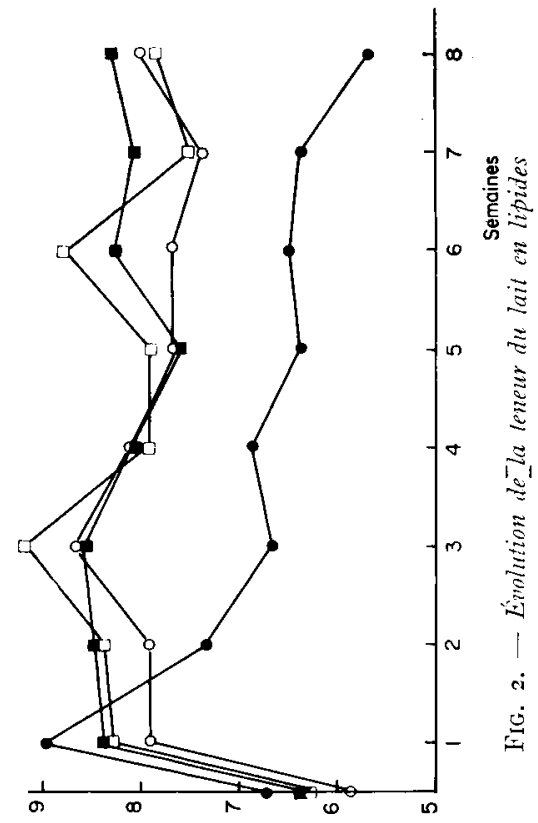

oold 6

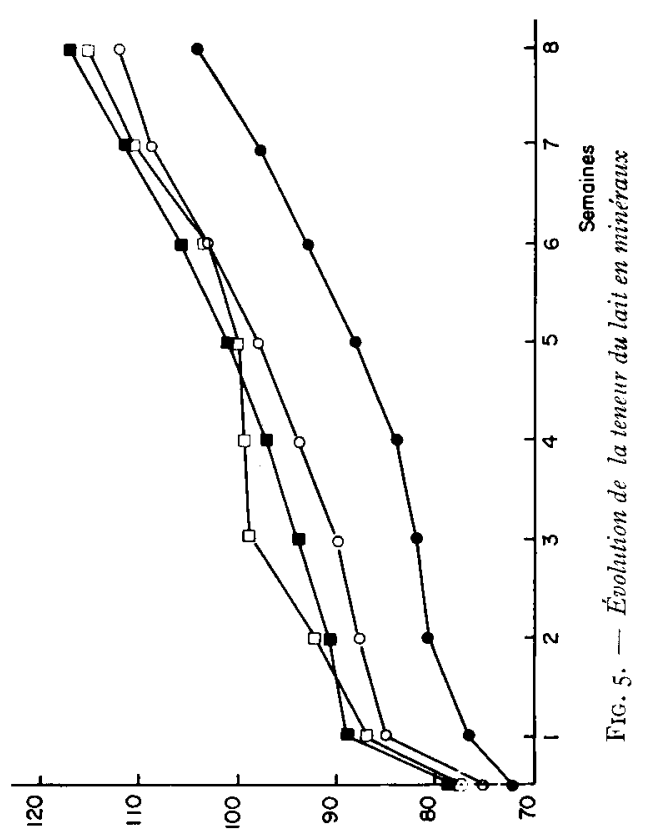

00001 d 5

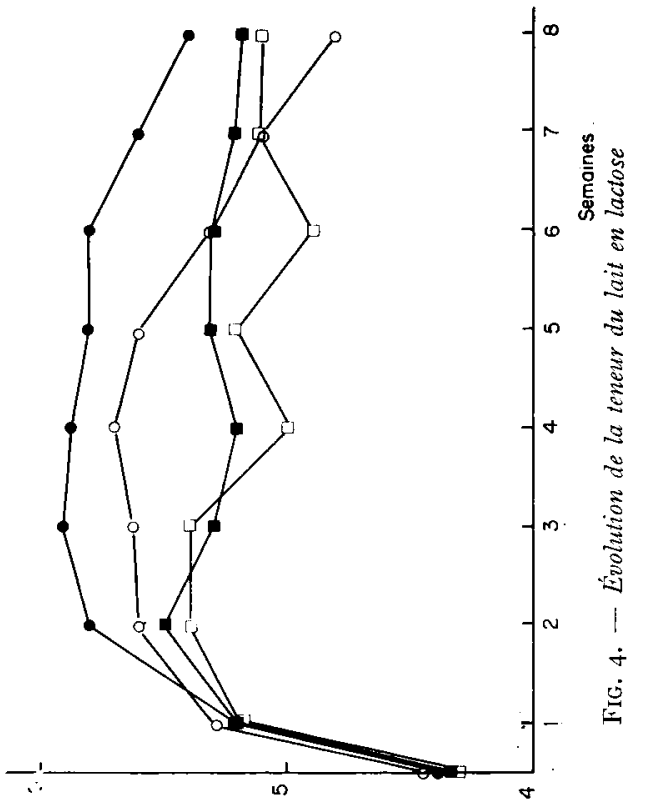

$001 \cdot 6$

Légende

$$
\begin{aligned}
& \text { Iot I } \\
& \text { Lot II } \\
& \text { Iot III } \\
& \text { Lot IV }
\end{aligned}
$$

Fig. 2 a 5 . Évolution des teneurs des principaux constituants du lait de truie au cours de la lactation 
Compte tenu des quantités de lait, les productions totales de chacun des principaux constituants s'établissent ainsi (tableau 4) :

\section{TABLEAU 4}

Quantités totales produites des principaux constituants du lait $(\mathrm{kg})$

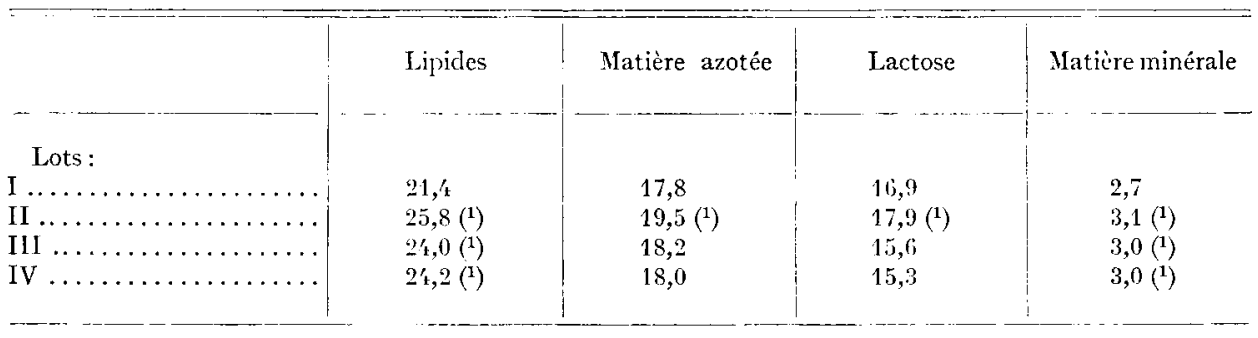

(1) Différence significative avec le lot 1

\section{$4^{\circ}$ Composition des lipides du lait}

Le tableau 5 rapporte les valeurs moyennes trouvées pour les indices d'iode, et la répartition des acides gras du lait des 4 lots de truies.

Ces résultats font apparaître dans tous les lots ayant reçu du saindoux, une augmentation notable des teneurs en acides gras insaturés. Cette augmentation porte sur tous les acides gras insaturés, mais plus spécialement sur l'acide linoléique (lot III).

\section{TABLEAU 5}

Répartition des acides gras du lait (1) ( $g$ p. $100 \mathrm{~g}$ lipides totaux)

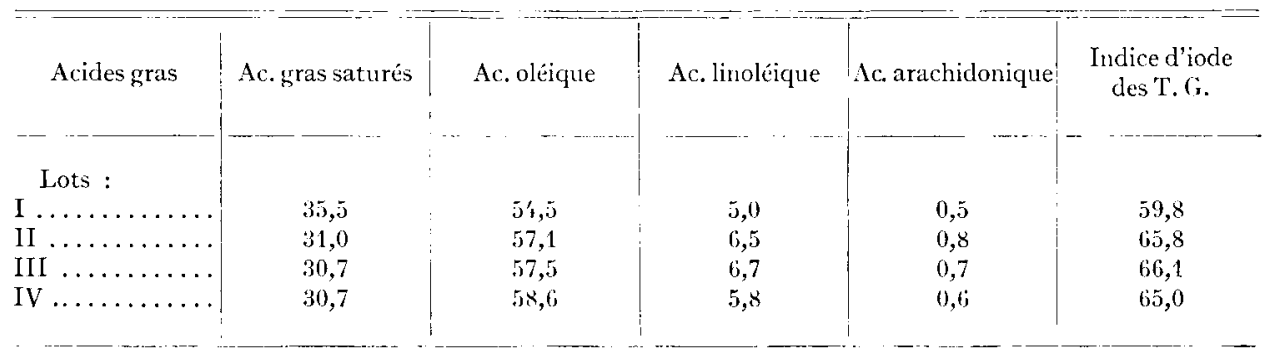

(1) Nous n'avons pas fait figrurer l'acide linolénique que nous n'avons trouvé qu'à l'état de traces.

\section{$5^{\circ}$ Variations corporelles}

Même dans les lots recevant de la graisse, les truies de notre expérience ont perdu du poids. Toutefois, ces pertes de poids et les variations de l'adiposité qui en ont résulté ont affecté différemment les truies de chaque lot, comme le montrent le tableau 6 et les figures 6 et 7 . 
Le lot témoin a perdu significativement plus de poids que les lots au saindoux II et III. Il semble qu'il en ait perdu plus également que le lot IV (même niveau azoté

TABLEAU 6

Variations corporelles des truies

\begin{tabular}{|c|c|c|c|}
\hline & \multirow{2}{*}{ Perte de poids $(\mathrm{kg})$} & \multicolumn{2}{|c|}{$\begin{array}{c}\text { Diminution de l'ćpaisseur du tissu } \\
\text { adipeux sous-cutané }\end{array}$} \\
\hline & & $\mathrm{mm}$ & $\begin{array}{l}\text { p. } 100 \text { de la valeur } \\
\text { à la parturition }\end{array}$ \\
\hline & $36,7 \ldots \quad 7,4$ & $5,4-2,7$ & 13 \\
\hline II $\ldots \ldots \ldots \ldots \ldots \ldots \ldots \ldots$ & $28,4=13,1$ & $1,0 \pm 4,1$ & 11 \\
\hline III $\ldots \ldots \ldots \ldots \ldots \ldots \ldots \ldots$ & $21,7 \div 7,1$ & $3,2 \pm 2,3$ & 11 \\
\hline IV $\ldots \ldots \ldots \ldots \ldots \ldots \ldots \ldots$ & $30,3=11,9$ & 8,0 士 2,8 & 21 \\
\hline
\end{tabular}

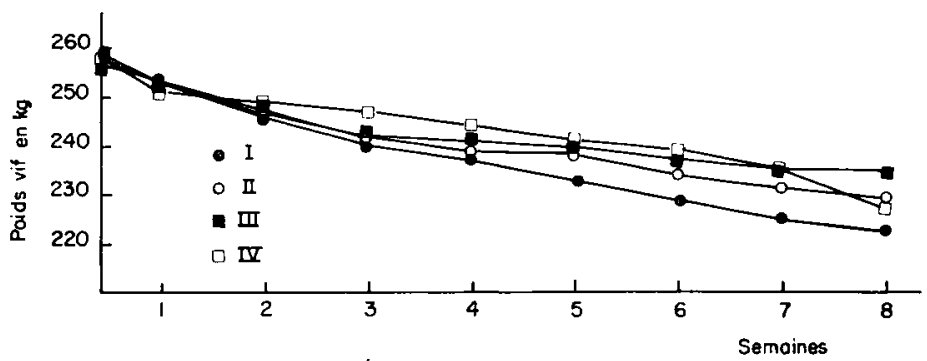

FIG. 6. - Evolution du poids des Truies

et énergétique), mais la différence n'est pas significative. On peut noter aussi chez ce dernier une perte de poids très sensible au cours de la dernière semaine de lactation (figure 6).

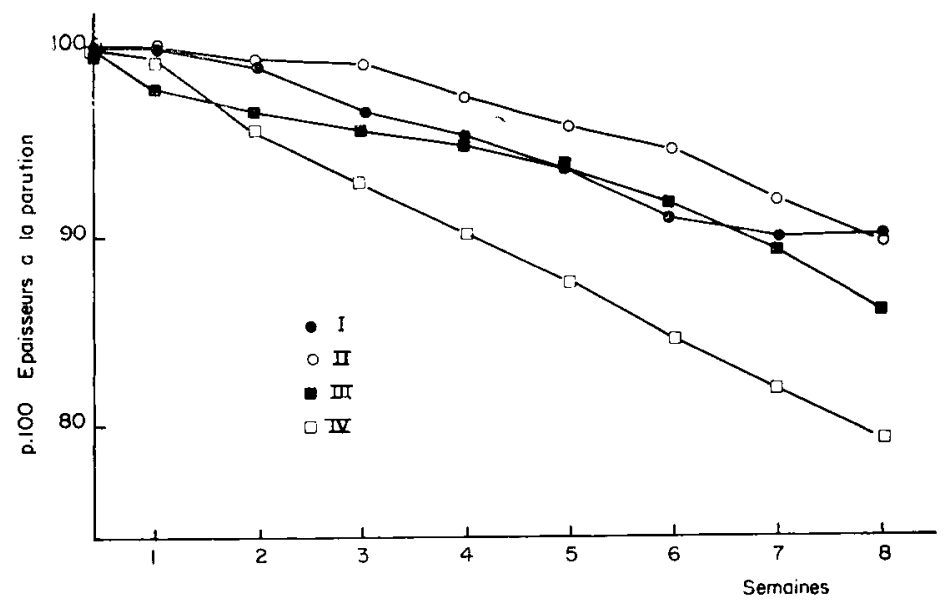

FIG. 7. - Evolution de l'éfaisseur du tissu adipeux sous-cutané dorsal (p. roo des valeurs à la parturition) 
La perte de lipides (diminution du tissu adipeux sous-cutané) a été sensiblement la même dans les 3 premiers lots et significativement plus importante dans le dernier lot. La figure 7 montre que ce phénomène s'est manifesté dès la première semaine de lactation.

\section{$6^{\circ}$ Croissance des porcelets}

Le tableau 7 rapporte les principales caractéristiques des portées de porcelets de chaque lot à 56 jours et la figure 8 l'évolution du poids moyen de ces porcelets au cours de la lactation. Les différences de poids à 56 jours entre le lot I et les autres lots sont significatives à $\mathrm{P}=0,05$.

\section{TABLEAU 7}

Portées au sevrage

\begin{tabular}{|c|c|c|c|}
\hline & $\begin{array}{l}\text { Nombre de porcelets } \\
\text { sevrés par portée }\end{array}$ & $\begin{array}{l}\text { Poids moyen } \\
\text { des porcelets à } \\
56 \mathrm{j}(\mathrm{kg})\end{array}$ & $\begin{array}{l}\text { Consommation d'ali- } \\
\text { ment complémentaire } \\
\text { par porcelet }(\mathrm{kg})\end{array}$ \\
\hline I $\ldots \ldots \ldots \ldots \ldots \ldots \ldots$ & 8,5 & $13,6 \pm 1,6$ & 5,6 \\
\hline II $\ldots \ldots \ldots \ldots \ldots \ldots \ldots \ldots$ & 8,5 & $15,8 \pm 0,8$ & 5,9 \\
\hline III $\ldots \ldots \ldots \ldots \ldots \ldots \ldots \ldots$ & 8,3 & $15,3 \pm 1,5$ & 5,7 \\
\hline IV $\ldots \ldots \ldots \ldots \ldots \ldots \ldots \ldots$ & 8,5 & $15,2 \pm 1,6$ & 5,6 \\
\hline
\end{tabular}

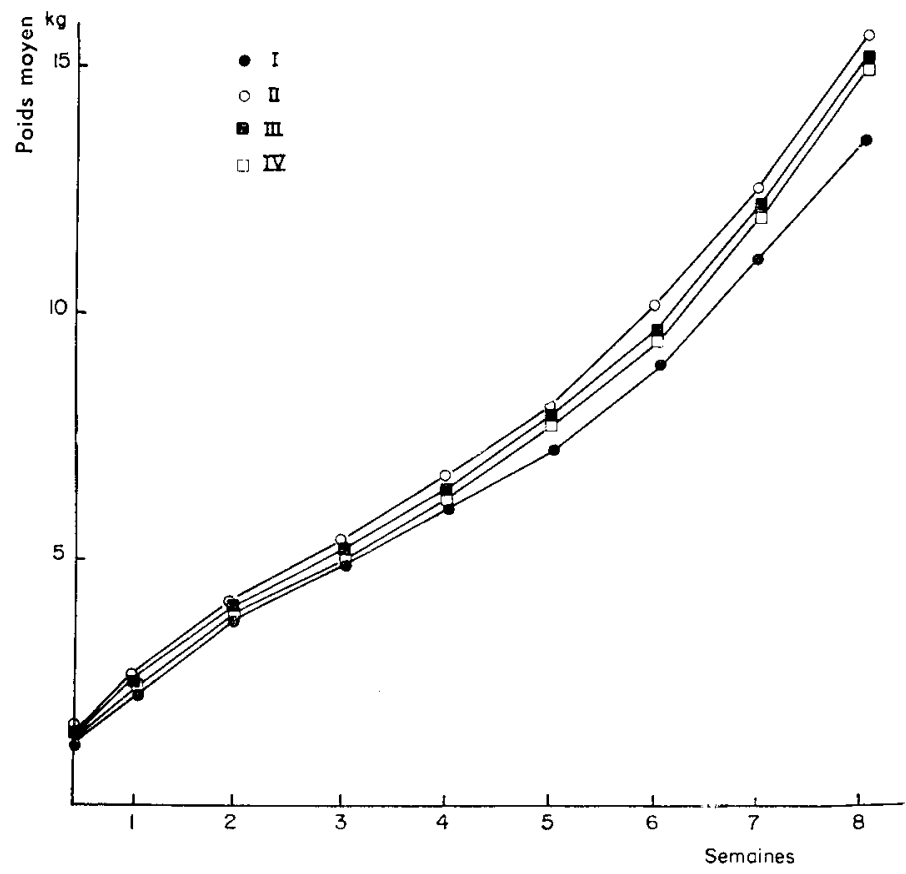

FIG. 8. - Courbes de croissance des porcelels de la naissance au sevrage Annales de Zootechnie. - I962. 
On remarque sur la figure 8 que la différence de vitesse de croissance entre les porcelets du lot I et ceux des autres lots apparaît surtout après la $3^{\mathrm{e}}$ semaine de lactation.

\section{DISCUSSION}

\section{Io Quantités de lait produites et composition du lait}

S'il était logique de s'attendre à une variation des quantités de constituants sécrétés dans le lait sous l'effet d'une augmentation de l'apport alimentaire (lots II et III), la façon dont ce résultat a été obtenu est un peu étonnante. Bien plus que l'augmentation de la quantité de lait dans le lot II (saindoux seul), le fait le plus important qui demeure, est l'augmentation du taux de sécrétion de la matière grasse dans le lait des trois lots qui recevaient des calories lipidiques. Ce phénomène prend d'ailleurs un relief particulier dans le lot IV pour lequel la seule modification du régime portait sur la nature des calories, l'apport total de nutriments étant le même que celui du lot témoin.

On ne peut manquer de trouver ici une différence avec ce qui se passe dans d'autres espèces, chez les Ruminants par exemple, où comme le rapportent JARRIGE et Journet (I959) l'effet de l'introduction de graisse dans la ration, lorsqu'il existe, porte plus sur la quantité de lait produite que sur une modification du taux butyreux (Loosl. et al., I944). Chez la Truie, au contraire, à l'exception de notre lot II, c'est le taux de matière grasse qui est modifié et non la quantité de lait, ce qui confirme nos résultats antérieurs (SALMON-LEGAGNEUR, Ig60) et a fait dire, en d'autres circonstances, à SHEFFY (I952) que le mécanisme de régulation de la sécrétion des lipides du lait, dans le cas d'une augmentation de l'apport alimentaire, ne serait pas le même chez ces diffétentes espèces. Bien qu'on ait signalé aussi chez des vaches laitières des cas analogues à ce que nous avons observé (ALLEN, I94I), l'explication d'un tel phénomène parâ̂t possible, si l'on admet que chez la Truie la graisse du régime peut participer plus ou moins directement à l'élaboration des lipides du lait, alors que chez les Ruminants la graisse ne serait utilisée en général qu'après des remaniements importants et n'aurait même parfois qu'un rôle énergétique banal.

Dans notre expérience en effet, comme le montre le tableau 4, seule la production de lipides du lait a été notablement modifiée. Tout au plus peut-on noter, à côté, une légère augmentation, difficilement explicable d'ailleurs, de la sécrétion des matières minérales ( + Io p. IOO), mais qui va de pair avec une très faible diminution de la teneur en lactose. Nous avons déjà eu l'occasion de souligner les relations étroites que l'on pouvait trouver chez la 'Truie entre les teneurs de certains constituants, comme le lactose, et les quantités de lait sécrété (SALMON-LEGAGNEUR, I96I a). I1 n'est donc pas étonnant ici que les quantités de lait aient peu varié, puisque la sécrétion du lactose n'a été elle-même que peu affectée.

L'évolution au cours de la lactation de la teneur du lait en matière grasse n'est pas sans intérêt. On peut en effet trouver deux interprétations au fait que l'effet de la matière grasse du régime n'est visible qu'après la fin de la première semaine (figure 2). On peut penser à une origine non alimentaire, importante, des lipides du 
lait au début de la lactation) par exemple à partir des lipides corporels, ce qui réduirait d'autant l'influence du régime (SALMON-LEGAGNEUR, I963). Ou bien, jusqu'à la fin de la première semaine, le niveau de la production est tel que les glucides de la ration suffisent à assurer la lipogenèse à son taux le plus élevé sans qu'un supplément de graisse soit capable d'améliorer ce taux. Par la suite, les lipides corporels peuvent faire défaut, ou le niveau de la production s'élever au-delà des possibilités des glucides : à ce moment, seuls les régimes supplémentés en graisse permettent le maintien de la secrétion des lipides du lait à un haut niveau.

La comparaison des différents lots recevant de la matière grasse fait aussi apparaitre quelques points intéressants : par exemple, le supplément de protéines alimentaires donné au lot III n'a eu que peu d'effet, mis à part une légère augmentation du taux de matière azotée du lait (tableau 5) qui ne s'est pas répercutée au niveau de la production, puisque cette dernière a baissé. Tout semble indiquer que le niveau énergie/protéine du lot II s'est montré plus favorable que celui des lots I, III et IV, ce qui signifierait que dans ces derniers les protéines sont en excès par rapport à l'énergie et qu'une partie de ces protéines est donc dégradée et mal utilisée, ce qui provoquerait une baisse de la production. Sur un plan analogue, les résultats du lot IV peuvent surprendre : ils montrent tout d'abord, qu'à taux d'énergie égal, les lipides semblent présenter une meilleure efficacité comme précurseurs et comme source d'énergie que les glucides pour la lipogenèse du lait, ce qui a été parfois contesté jusqu'à présent (FRENCH, I952), Mais il est curieux de constater que l'amélioration qui en a résulté est la même que celle des rations plus abondantes contenant elles-mêmes des lipides, comme celles des lots II et III. Ceci semblerait indiquer que, quel que soit le niveau énergétique de la ration, les graisses du régime sont utilisées en priorité par la mamelle. Toutefois, ce phénomène reçoit plus loin une autre explication, compte tenu des variations corporelles des truies, car les truies du lot IV se délipident plus que les autres au cours de la lactation.

\section{$2^{\circ}$ Composition en acides gras}

L'examen du tableau 5 montre bien que la modification subie par les lipides du lait dans les lots supplémentés en saindoux n'a pas été seulement quantitative, mais aussi qualitative. Ceci constitue une nouvelle peuve du passage plus ou moins direct de certains acides gras du régime dans le lait : l'enrichissement relatif des lipides du lait en acide linoléique ne peut s'expliquer que si celui-ci a été prélevé dans le régime, puisque l'animal n'en synthétise normalement pas. Il est vraisemblable que l'augmentation de la teneur en acides oléique et arachidonique procède du même mécanisme. Ce phénomènen'est pas nouveau, il a déjà été signalé à des degrés divers dans d'autres espèces, notamment chez la Vache (KUzDzaI-SAvore, I959), chez la Ratte (BEARE, I96I) et chez la Femme (Insuli, I959), mais jamais encore chez la Truie.

Mais un fait demeure curieux dans notre expérience, c'est que les teneurs des différents acides gras insaturés dans le lait deviennent supérieures à celles dans la matière grasse de départ $\left({ }^{1}\right)$. Un tel phénomène avait déjà été rencontré par FEVRIER (r959) sur des porcs en croissance. On peut avancer plusieurs hypothèses pour l'ex-

(1) Le saindoux que nous avons utilisé avait la composition suivante : ac. saturés : 37,9 p. 1oo, ac. oléique:

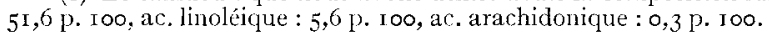


pliquer. Tout d'abord, on peut penser que seule la fraction la plus insaturée des graisses dont une partie n'est pas synthétisée par l'animal, est affectée par ce phénomène et qu'il en résulte une sorte de concentration de cette fraction dans le lait, avec corrélativement une diminution de la fraction saturée. On peut imaginer aussi, qu'au cours de l'utilisation métabolique des graisses, et notamment du saindoux, il survient certaines modifications de structure et que des glycérides ou des acides gras insaturés soient mis en plus grand nombre à la disposition de la mamelle et utilisés en priorité Mais il faut retenir aussi que, quel que soit le mécanisme envisagé, la composition des graisses du lait de Truie a tendance à se rapprocher de celle des dépôts adipeux maternels, plus insaturés que le saindoux de notre expérience (SAr.MoNLEGAGNEUR, Ig63). Ceci montre que la lipogenèse du lait et celle des dépôts procèdent des mêmes phénomènes chez cette espèce et qu'il $\mathrm{y}$ a au cours de la lactation des interrelations entre la graisse du régime, la graisse des dépôts et les lipides du lait, qui rendent ce problème particulièrement complexe.

\section{$3^{\circ}$ lariations corporelles des truies}

Le fait que les truies supplémentées en saindoux aient perdu moins de poids au cours de la lactation que les truies témoins, peut s'interpréter comme l'indication d'une intervention des graisses du régime sur cette perte de poids. Ceci semblerait montrer que la carence alimentaire supportée habituellement par la truie en lactation est essentiellement énergétique, ce que confirme l'importance de la mobilisation des dépôts adipeux.

Il faut toutefois remarquer que la variation de poids la plus faible a été enregistrée dans le lot III qui recevait, en plus de la graisse, un supplément de protéines. Ceci indique bien que ces dernières excercent également un effet protecteur vis-à-vis de la perte de poids et que, dans certains cas, une mobilisation des protéines corporelles peut s'effectuer au bénéfice de la lactation. Que ces protéines soient utilisées en tant que source d'azote ou d'énergie, c'est ce que nous ne savons pas encore. Mais il est bien certain qu'une augmentation du niveau énergétique seul de la ration, ne suffit pas pour arrêter le catabolisme de lactation.

Il n'est pas sans intérêt de souligner que dans le lot IV, qui recevait la même quantité d'énergie et d'azote que le lot I, l'effet de protection des lipides sur la perte de poids a été moins net que dans les autres lots. On remarque également que la mobilisation du tissu adipeux a été par contre beaucoup plus importante que dans les autres lots, ce qui entraînerait par déduction un catabolisme azoté moins important. Dans ce lot donc, la présence de graisse dans la ration aurait eu un effet d'épargne plus sensible sur les protéines tissulaires que sur les graisses de réserve. Il y a une explication à ce phénomène : l'augmentation de la sécrétion des lipides dans le lait, aussi importante dans ce lot que dans les lots II et III, n'a pu se faire, sans supplément alimentaire, qu'au prix d'une mobilisation plus importante des tissus de réserve. Ou, ce qui revient au même, toutes les graisses du régime ont été utilisées pour la lipogenèse du lait, alors que dans les autres groupes une partie de ces graisses participait au maintien des réserves corporelles. Comme le montre la figure 6 , ceci s'est passé surtout au cours des dernières semaines de lactation. Il est vraisemblable que, dans ce cas, les graisses corporelles ont participé, directement ou non, à la lipogenèse 
du lait. Nous trouvons ici un nouvel exemple des relations qui se créent chez la truie entre l'aliment, les dépôts et le lait.

\section{$4^{\circ}$ Croissance des porcelets}

Il est manifeste que la présence de saindoux dans l'alimentation de la truie a en pour effet secondaire une amélioration de la croissance des porcelets, et plus spécialement du poids au sevrage, que l'on peut évaluer suivant les cas à II à I $6 \mathrm{p}$. Ioo de la performance des témoins.

Doit-on voir ici une influence spécifique du saindoux, ou plus simplement la conséquence d'une augmentation de l'ingéré calorique des porcelets? I1 est probable que la seconde hypothèse est la plus valable. Certes, les régimes contenant de la graisse ont provoqué un certain enrichissement du lait en acides gras indispensables (ac. linoléique), mais le taux de cet acide dans le lait des animaux témoins, paraissait déjà élevé et sans doute suffisant pour qu'on ne puisse invoquer une stimulation de croissance analogue à celle observée par DEUEL (I956) sur des ratons dont les mères avaient été supplémentées en linoléate.

Par contre, une étude antérieure (SAIMON-LEGAGNEUR et AuMAITRE, Ig62) nous avait montré que 1'ingestion d'énergie était 1'un des facteurs importants de la vitesse de croissance pendant 1'allaitement et qu'il y avait une relation entre la quantité de lipides apportés par le lait et le poids du porcelet à différents stades. Le fait que dans notre expérience l'influence des régimes lipidiques apparaisse surtout après la $3^{\mathrm{e}}$ semaine de lactation, moment où les besoins totaux du porcelet augmentent considérablement, renforce cette interprétation.

On peut se demander toutefois si la supplémentation du régime maternel en graisse peut être considérée comme un moyen efficace d'améliorer la croissance des porcelets? L'amélioration constatée est, au demeurant, assez faible et un rapide calcul économique montre qu'elle est à la limite de la rentabilité. D'autres expériences avec des graisses et des taux différents sont sans doute nécessaires pour pouvoir conclure objectivement sur ce point.

\section{CONCLUSION}

Le premier effet de l'introduction de $\mathrm{r} 7 \mathrm{p}$. Ioo de saindoux dans le régime alimentaire de truies en lactation a été une augmentation de la sécrétion des lipides du lait. A une exception près, cette augmentation n'a pas été le résultat de la production d'une plus grande quantité de lait, mais, au contraire, d'une élévation de la teneur du lait en graisse. Cet enrichissement, relativement important (I5 p. IOo), du lait apparaît même dans le cas d'un régime iso-énergétique et isoazoté. La teneur des autres constituants du lait, sauf peut-être celle des matières minérales, n'est pas modifiée.

Corrélativement, la modification de la teneur en matière grasse s'accompagne d'un changement dans la répartition des acides gras et notamment d'un enrichissement en acides insaturés (oléique, linoléique et arachidonique), que l'on peut interprêter comme l'indication d'un passage plus ou moins direct de ces acides de l'aliment dans le lait. 
On note aussi une diminution plus ou moins prononcée (suivant le niveau énergétique), de la perte de poids maternelle au cours de la lactation, qui atteint son plein effet lorsque les truies reçoivent également un supplément protéique. L'intervention des graisses alimentaires dans la lipogenèse du lait et la mobilisation du tissu adipeux au cours de la lactation est ainsi clairement démontrée.

On observe subsidiairement une augmentation du poids au sevrage des porcelets, mais qui ne paraît pas suffisante à elle seule dans nos conditions pour justifier l'intérêt économique de cette pratique.

Reçu pour publication en décembre 1962.

\author{
SUMMARY \\ THE EFFECT OF SUPPLEMENTARY FAT (LARD) IN THE DIET UPON \\ MILK PRODUCTION IN SOWS
}

Fourty sows were alloted in four groups, each receiving one of the following diets : I Basal ; II Basal $+\mathrm{r} 7 \mathrm{p}$. roo lard ; III Basal $+\mathrm{r} 7 \mathrm{P}$. I00 lard $+3 \mathrm{p}$. Ioo fish meal; IV the same as III but fed at $80 \mathrm{p}$. roo level.

Digestibilities for both nitrogen and energy were determined by chromium oxide technique and it appears that diets I and IV were isocaloric and that diets I, III and IV had the same digestible nitrogen content.

The amount of milk produced was the same in all groups except II, whereas the milk fat percentage was increased by 15 p. 100 in the three groups receiving lard. Furthermore, the fatty acid composition of milk was also modified : oleic and linoleic acids increased and saturated acids decreased in groups II, III, IV. So it seems that the fat intake may be used directly by the sow for milk lipogenesis, but with some differences as compared to other species. This point is discussed.

On the other hand, losses in weight during lactation decreased in sows receiving fat and the weaning weight of piglets slightly increased.

\title{
RÉFÉRENCES BIBLIOGRAPHIQUES
}

Alles N., Fitci J. B., r94I. The influence of sustained high fat intake upon milk production. J. Dairy Sci., 24, 516-5!7.

Beare J. L., Gregory E. R., Smith D. M., Campbell J. A., I96I. The effect of rapeseed oil on reproduction and on the composition of rat milk fat. Canad. J. Biochen. Physiol., 39, 195-20I.

Deuel H. J., Martin C. R., Alfin-Slater R. B., i956. The effect of fat level of the diet on general nutrition. XII. The requirement of essential fatty acids for pregnancy and lactation. J. Nutr., 54, I93-199.

Duncan D. L., LodGE G. A., 1960. Diet in relation to reproduction and the viability of the young. III. Pigs. Commonoealth Bureau Anim. Nutr. Tech. Comm. 21, to6 p.

French C. E., Ingram R. H., Knoebel L. W., Swift R. W., i952. The influence of dietary fat and C.H. on reproduction and lactation in rats. J. Nuir., 48, 9 1 -1oz.

Insull W., Hirsch J., James T., Airens E. H., I959. 'The fatty acids of human milk. II. Alterations produced by manipulation of caloric balance and exchange of dietary fats. J. Clin. invesitgat., 38, 443-45०.

JARRIGE R., Journet M., I959. Influence des facteurs alimentaires et climatiques sur la teneur en matière grasse du lait. Ann. Nutr. Alim., 13, 233-278.

Kuzdzal-Savole S., I959. Influence de l'alimentation et du climat sur la matière grasse du lait de vache. Aspect qualitatif. Ann. Nutr. Alim., 13, 207-232.

LODGE G. A., 1959. The energy requirement of lactating sows and the influence of level of food intake upon milk production and reproduction performance. J. Agric. Sci., 53, I 77-191.

LODGE G. A., I962. The nutrition of the lactating sow, in Morgan J. T., et Lewis D. Nutrition of pigs and poultry, 224-237, Butterworths, London. 
Loosli J. K', Maynard L. A., Lucas H. L., I944. Further studies of the influence of different levels of fat intake upon milk secretion. Cornell Agr. Exp. Stat., Mémoir 265, 3I p., University ed. New York.

Norman R., Sheffy B. E., Willman J. P., i955. The effect of supplementary fat in the rations of lactating swine. J. Anim., Sci., 14, I 2 г9.

Paquot C., Mercier J., Mathieu A., Lefort D., Perron R., ig62. Les méthodes analytiques des lipides simples. Cahiers Techn. C. N.C.E.R. N. A., VIII, 28 I p., C. N. R. S. ed., Paris.

Raymond W. F., Minson D. J., I955. The use of chromic oxide for estimating the faecal production of grazing animals. J. Brit. Grassl. Soc., 10, 282-295.

Salmon-Legagneur E., 1957. Consommation alimentaire et production laitière chez la Truie. Résumé comm. $4^{\mathrm{e}}$ Congr. Int. Nutrit., 29, Paris.

Salmon-Legagneur E., i959 a. Description et utilisation d'une machine à traire les Truies. Ann. Zootech., 8, $349-356$.

Salmon-Legagneur E., i959 b. La composition du lait de Truie. Premières observations sur quelques facteurs de variation. Ann. Zootech., 8, 93-І工2.

Salmon-Legagneur E., i960. in Commission du lait du C. N. C. E. R. N. A, la production du lait. Ann. Nutr. Alim., 14, x-99.

Salmon-Legagneur E., ig6r $a$. La composition du lait de Truie. Relations entre les variations des teneurs du lactose et des autres constituants. Ann. Biol. anim. Bioch. Biophys., 1, 295-303.

Salmon-Legagieur E., Jacquot R., I 66 I $b$. Quelques aspects des relations nutritionnelles entre la gestation et la lactation chez la Truie. C. R. Acad. Sci. Paris, 253, I6r8-1620.

Salmon-Legagneur E., Aumaitre A., 1962. Influence de la quantité de lait et de sa composition sur la croissance du porcelet sous la mère. Ann. Zootech. 11, 18r-196.

Salmon-Legagneur E., Ig63. Relations entre les graisses du régime, les lipides corporels et les lipides du lait chez la Truie. C. R. Acad. Sci. Paris, 256, I 372.

ShefFy B. E., Phillips P. H., Dymsza H. A., Grummer R. H., Bohstedt G., I952. Fat, fat constants and phospholipid content of sow's milk. J. Anim. Sci., 2, 727-735.

Widlett E. L., Maruyama C., ig46. The effect of intake of garbage fat upon fat content of sow's milk. J. Anim., Sci., 5, 365-370. 